\section{nephron \\ Clinical}

Practice
Nephron 2019;143:28-32

DOI: 10.1159/000494694
Received: June 26, 2018

Accepted after revision: October 18, 2018

Published online: January 9, 2019

\title{
Underlying Mechanisms of Renal Lipotoxicity in Obesity
}

\author{
Elia Escasany Adriana Izquierdo-Lahuerta Gema Medina-Gomez
}

Área de Bioquímica y Biología Molecular, Departamento de Ciencias Básicas de la Salud, Facultad de Ciencias de la Salud, Universidad Rey Juan Carlos, Alcorcón, Madrid, Spain

\section{Keywords}

Obesity · Adipose tissue expandability · Lipotoxicity · Peroxisome proliferator-activated receptor gamma .

Chronic kidney disease

\begin{abstract}
The recent and ongoing worldwide increase in the prevalence of obesity parallels the increase in the incidence of chronic kidney disease (CKD). This association suggests an implication of lipotoxicity in the development of kidney diseases. The increased influx of lipids into the kidney can be explained in the context of the "Adipose Tissue Expandability Hypothesis". This hypothesis states that the adipose tissue has a limited expansion capability, which is different for each individual, and once this limit is reached, the adipose tissue cannot store any more lipids and will thus release them into the bloodstream. The accumulation of lipids in the kidney is known as renal lipotoxicity. Renal lipo-
\end{abstract}

toxicity is known to cause detrimental effects on the kidney by several mechanisms of action including reclusion of proinflammatory factors, oxidative and ER stress development, insulin resistance (IR), lipid metabolism deregulation or renin-angiotensin aldosterone system overactivation. Isoform peroxisome proliferator-activated receptor gamma (PPARY) seems to play an important role in the development of this lipotoxicity as proven by several studies in animals and cultured cells. Thus, PPARy agonists are of interest in the therapeutic approach to treat CKD in the context of obesity. This review aims to summarize our current knowledge of the mechanism by which lipotoxicity affects renal structure and function using in vivo and in vitro models as examples focusing on PPARy.

두 2019 S. Karger AG, Basel

Contribution from the CME Course of the DIABESITY Working Group of the ERA-EDTA, Lisbon, November 24-25, 2017.

\section{KARGER}

(C) 2019 S. Karger AG, Basel

E-Mail karger@karger.com

www.karger.com/nef
Gema Medina-Gomez

Área de Bioquímica y Biología Molecular, Departamento de Ciencias Básicas de la Salud Facultad de Ciencias de la Salud, Universidad Rey Juan Carlos

Avendia de Atenas s/n, Alcorcón ES-28922 Madrid (Spain)

E-Mail gema.medina@urjc.es 


\section{Introduction}

Obesity is considered a worldwide epidemic affecting more than 600 million adults and up to $17 \%$ among children and youth. Moreover, its prevalence has been predicted to grow by $40 \%$ in the next decade. The development of obesity requires a state of positive energy balance with increased ingestion of fat and decreased energy expenditure, leading thus to fat deposition. In order to store all the surplus of calories, the adipose tissue needs to expand by either generating more adipocytes (hyperplasia) or enlarging the already existing adipocytes (hypertrophy). However, the adipose tissue expansion capacity is not infinite and once the limit is reached, fat will start to leak and accumulate ectopically, as stated in the "Adipose Tissue Expandability Hypothesis". The ectopic accumulation of lipids in different organs contributes to their damage through toxic processes named lipotoxicity [1]. When the muscle and the liver are affected by the deposition of fat, they become less responsive to insulin and thus, the pancreas tries to compensate this by excreting more insulin. If this situation is maintained in the time, the pancreas is unable to compensate and its normal function is affected. This is how lipotoxicity is linked to the development of insulin resistance (IR) and, in the long term, to the development of type 2 diabetes mellitus. Lipids can also accumulate in the muscle, liver, brain, placenta, heart and, of relevance for this review, in the kidney causing detrimental effects.

\section{Renal Lipotoxicity: Mechanisms of Action}

Fatty kidney is a term that has recently started to be used to address the pathological situation with lipid deposition in the kidney. The term appears as a replicate of the well-known term fatty liver [2]. Fatty kidneys show common characteristics that are a result of the detrimental effects that lipids exert. Several mechanisms of action by which lipids can cause kidney damage have been proposed.

\section{Adipokines and Pro-inflammatory Factors}

During obesity, the adipose tissue suffers an increment in its endocrine activity, which leads to a deregulation in the production and release to the bloodstream of adipokines such as leptin, resistin and adiponectin. The latter is produced in decreased levels, while leptin and resistin levels are increased. Adipokines are involved in kidney damage through the mediating endothelial dysfunction,

Obesity and Renal Lipotoxicity inducing oxidative stress and inflammation as well as stimulating the renal sympathetic nervous activity $[3,4]$.

At the same time, the adipose tissue increases the secretion of pro-inflammatory factors during obesity such as macrophage chemoattractant protein-1 (MCP-1), macrophage migration inhibition factor (MIF), chemokine (C-C motif) ligand 5 (CCL5) and macrophage colony stimulating factor, which recruit macrophages. Indeed, it has been observed that treatment with anti-macrophage migration inhibition factor- 1 antibodies reduces leukocyte infiltration into the kidney, proteinuria and renal loss in a rat model [5].

\section{Oxidative Stress, Fibrosis and ER Stress}

Due to the massive recruitment of inflammatory cells to the kidney, the uptake of oxygen in the area increases, thereby leading to an increment in the production of reactive oxygen species (ROS). ROS may interfere with renal tubule ion transport by altering the renal and blood pressure while causing oxidative injury to proximal tubules. ROS may also induce the expression of profibrotic molecules such as transforming growth factor-beta 1 (TGF- $\beta 1$ ) and plasminogen activator inhibitor-1 (PAI-1), through the nuclear factor kappa-light-chain-enhancer of activated $\mathrm{B}(\mathrm{NF}-\kappa \mathrm{B})$ cells and nicotinamide adenine dinucleotide phosphate oxidase (NADPH) pathways [6], therefore playing a major role in the development of renal fibrosis.

Oxidative stress may also contribute to the development of ER stress by promoting protein unfolding and activating the unfolded protein response. It has been shown that dietary saturated free fatty acids induce ER stress in kidneys of animal models [7] and in renal culture cells [8]. In fact, the treatment with palmitic acid promotes oxidative and ER stress [9].

\section{Insulin Resistance}

IR affects the kidney and especially the podocytes. Podocytes are very specialized and terminally differentiated epithelial cells, which like neurons, do not divide. Thus, podocyte loss is an irreversible event. Podocytes are 1 of the 3 components of the glomerular filtration barrier (GFB), whose role is to allow the selective filtration of molecules. Loss of podocytes leaves the GFB partially denuded allowing leaking of several macromolecules leading to proteinuria. Podocytes are the most sensitive renal cells to insulin and thus the IR associated to obesity largely affects them. Indeed, studies performed in cultured podocytes have shown that treatment with palmitic acid promotes IR and changes in the cytoskeleton leading to apoptosis [9]. 
Renin-Angiotensin Aldosterone System Axis

Importantly for the kidney, the adipose tissue secretes all components of the renin-angiotensin aldosterone system (RAAS). During obesity, there is an overactivation of this axis that may translate into hyperfiltration. Under this situation, the glomerulus manifests a big physical stress and, consequential damages for the GFB, especially the podocytes [10]. Loss of podocytes is a chain reaction that ends up with the loss of the entire glomerulus and thus nephrons. This might translate into a decreased glomerular filtration rate (GFR). Values of GFR under $60 \%$ are considered pathological. If sustained for a long time, patients are at risk of entering in chronic kidney disease (CKD) and finally end-stage renal disease, which requires dialysis and supposes a major risk factor of cardiovascular events and death [11].

\section{Renal Lipid Metabolism and Lipid Droplets}

Lipotoxicity may affect renal lipid metabolism leading to an increase in the proteins involved in lipid synthesis, while decreasing those in charge of oxidation of lipids. When excess fatty acids are not oxidized, they are esterified with glycerol and become lipid droplets. Therefore, it is very common in obese patients to find lipid droplets inside renal cells, especially podocytes. This metabolic deregulation exacerbates the renal lipid deposition, leading to a state of energy depletion that finally causes cell apoptosis and contributes to CKD [12].

\section{Peroxisome Proliferator-Activated Receptor Gamma and Renal Lipotoxicity}

Peroxisome proliferator-activated receptor gamma $(\mathrm{PPAR} \gamma)$ is a nuclear receptor critically involved in adipogenesis, glucose metabolism, triglyceride storage and energy homeostasis. Three isoforms of this protein are found in humans: PPAR $\gamma 1$, which is widely distributed at low levels; PPAR $\gamma 2$, with an exclusive expression in the adipose tissue, except in response to overnutrition, which it expresses in other organs; and PPAR $\gamma 3$, which appears to be limited to white adipocytes. It has been shown that PPAR $\gamma$ is expressed in different regions of the kidney including collecting ducts, medullary interstitial cells, the juxtaglomerular apparatus and the glomeruli.

Mutations in the PPAR $\gamma$ gene lead to dysfunctional lipid and glucose homeostasis and have been directly related to type 2 diabetes mellitus and obesity [13] and familial partial lipodystrophy type 3 (OMIM604367).
In animal models, it has been shown that mice deficient in PPAR $\gamma$ present alterations in renal lipid metabolism. The specific PPAR $\gamma 2 \mathrm{KO}$ mouse model shows a tendency towards but not a well-established kidney disease. Based on these results, the POKO mouse, generated by ablation of the PPAR $\gamma 2$ isoform in a leptin-deficient obese $(o b / o b)$ background, represents a model of the metabolic syndrome [14]. POKO mice are more insulin resistant as early as 4 weeks old than $o b / o b$ mice despite weighting less than half the weight of an $o b / o b$ mouse. POKO mice show accelerated renal damage with dyslipidaemia, altered glucose and lipid renal metabolism, lipid droplets deposition in glomeruli, hypertension and albuminuria [15]. Also, it seems that mice lacking macrophage-specific expression of PPAR $\gamma$ develop glomerulonephritis and autoantibodies to nuclear antigens, resembling the nephritis seen in systemic lupus erythematosus with tubule-interstitial lipid deposition that leads to lipidmediated renal damage [16]. Moreover, low adiponectin, which is an adipokine secreted by adipose tissue and regulated by PPAR $\gamma$ levels, correlates with albuminuria in obese patients [17]. All these findings highlight the crucial role that PPAR $\gamma$ may have in the maintenance of renal homeostasis [18].

\section{PPARy Agonists as Therapy in Renal Injury}

Importantly, Thiazolidinediones (TZDs), synthetic PPAR $\gamma$ agonists, have been shown to have reno-protective effects (Fig. 1). Indeed, TZDs decrease proteinuria independent of glycaemic control and are also thought to be mitochondrial-protective, thereby inhibiting renal inflammation and oxidative stress. TZDs show antiinflammatory effects by diminishing nuclear factor kappa-light-chain-enhancer of activated B cells, tumour necrosis factor $\alpha$ and interleukin 6 , as well as anti-fibrotic effects being able to even reverse the epithelial-mesenchymal transition in cultured human cells [19]. On the other hand, some TZDs have shown vasodilating properties in both the afferent and efferent arterioles of rabbit kidney, thereby decreasing the glomerular capillary pressure [20].

Unfortunately, the use of this group of agonists is greatly restricted due to their severe side effects, which include bone loss, oedema, fluid retention and thus, increased cardiovascular risk. Huge efforts are being made to optimize lower doses of TZD or the use of endogenous PPAR $\gamma$ agonists. Telmisartan, a renin-angiotensin aldosterone system blocker that also acts as a partial PPAR $\gamma$
Escasany/Izquierdo-Lahuerta/MedinaGomez 
Fig. 1. Renal lipotoxicity and PPAR $\gamma$ agonists effects. The lipotoxic effects caused by the ectopic accumulation of lipids in the kidney include oxidative stress, fibrosis, ER stress, inflammation, IR in the podocytes and RAAS overactivation, which translates into hyperfiltration and leads to podocyte loss and proteinuria. All these detrimental effects can be reversed by PPAR $\gamma$ agonist improving renal functioning during obesity. PPAR $\gamma$, peroxisome proliferator-activated receptor gamma; ROS, reactive oxygen species; IR, insulin resistance; RAAS, renin-angiotensin aldosterone system.

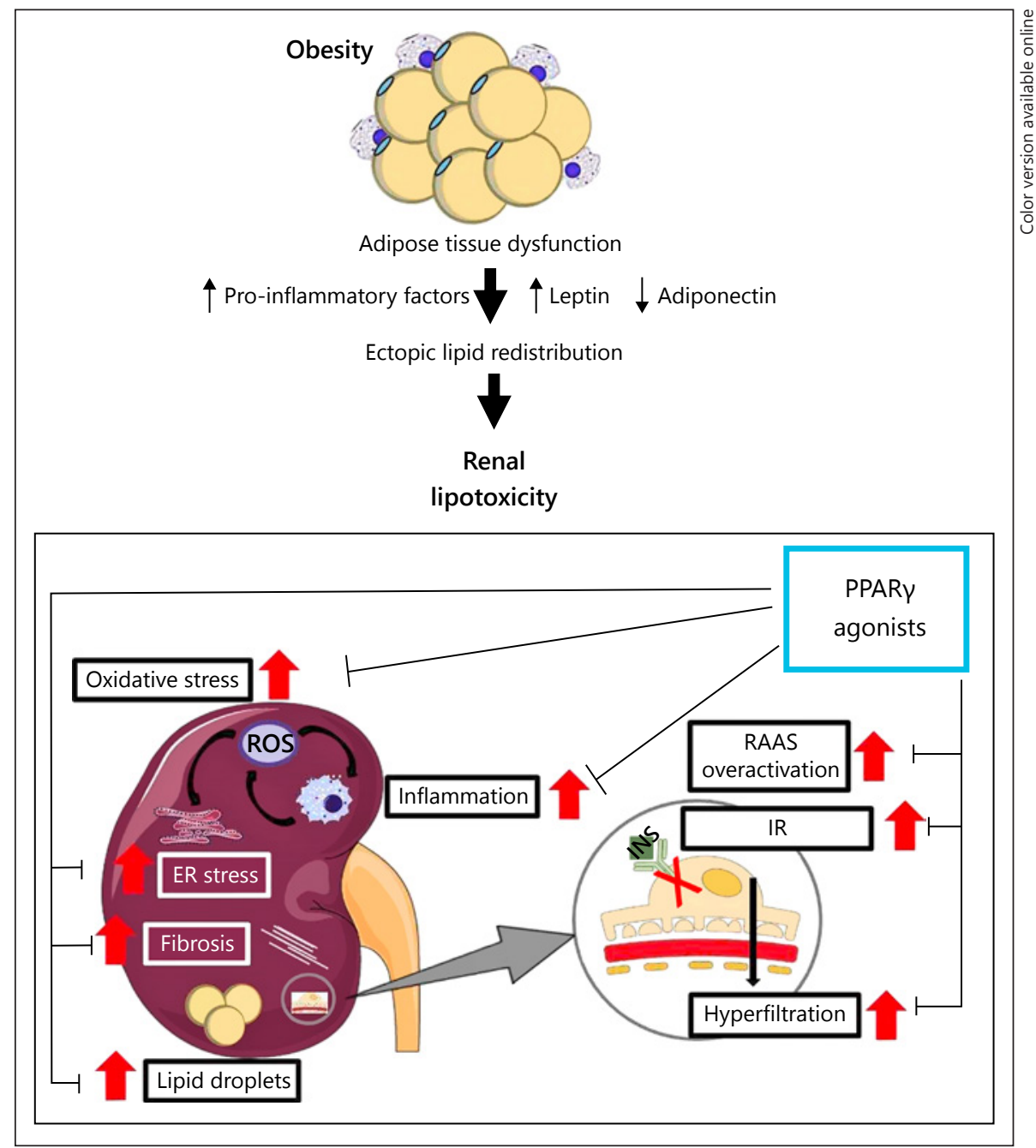

agonist is a relatively new therapy that holds promise [21]. By activating PPAR $\gamma$, telmisartan may decrease proteinuria and inflammation, consequently conferring reno-protection.

\section{Conclusions}

Obesity is a condition that is continuing to affect a great number of people worldwide and the incidence of $\mathrm{CKD}$ is expected to rise. Since renal disease is largely irreversible, especially when podocytes start to get affected by this lipid deposition, it is of extreme importance to unravel the early steps before patients enter CKD or end-stage renal disease. We believe that unravelling the specific effects of lipotoxicity at renal level could open new avenues of research for the discovery of early diagnosis and therapeutic strategies.
Likewise, a better understanding of the molecular mechanisms of action of PPAR $\gamma$ in the kidney may allow the development of new agonists and modulators to improve the management of kidney disease without side effects.

\section{Acknowledgement}

Research conducted for this publication was supported by Ministerio de Economía y Competitividad (BFU2013-47384-R and BFU2016-78951-R) and Comunidad de Madrid (S2010/ BMD-2423 and B2017BMD-368), Spain.

\section{Disclosure Statement}

The authors declare that they have no conflicts of interest to disclose. 


\section{References}

1 Virtue S, Vidal-Puig A: Adipose tissue expandability, lipotoxicity and the metabolic syndrome - an allostatic perspective. Biochim Biophys Acta 2010;1801:338-349.

2 de Vries AP, Ruggenenti P, Ruan XZ, Praga M, Cruzado JM, Bajema IM, et al: Fatty kidney: emerging role of ectopic lipid in obesityrelated renal disease. Lancet Diabetes Endocrinol 2014;2:417-426.

3 Rüster C, Wolf G: Adipokines promote chronic kidney disease. Nephrol Dial Transplant 2013;28(suppl 4):iv8-iv14.

4 Zhu Q, Scherer PE: Immunologic and endocrine functions of adipose tissue: implications for kidney disease. Nat Rev Nephrol 2018;14: 105-120.

5 Lan HY, Bacher M, Yang N, Mu W, NikolicPaterson DJ, Metz C, et al: The pathogenic role of macrophage migration inhibitory factor in immunologically induced kidney disease in the rat. J Exp Med 1997;185:14551465.

6 Welsh GI, Hale LJ, Eremina V, Jeansson M, Maezawa Y, Lennon R, et al: Insulin signaling to the glomerular podocyte is critical for normal kidney function. Cell Metab 2010;12: 329-340

7 Li C, Lin Y, Luo R, Chen S, Wang F, Zheng P, et al: Intrarenal renin-angiotensin system mediates fatty acid-induced ER stress in the kidney. Am J Physiol Renal Physiol 2016;310: F351-F363.

8 Sieber J, Lindenmeyer MT, Kampe K, Campbell KN, Cohen CD, Hopfer H, et al: Regula- tion of podocyte survival and endoplasmic reticulum stress by fatty acids. Am J Physiol Renal Physiol 2010;299:F821-F829.

9 Martínez-García C, Izquierdo-Lahuerta A, Vivas Y, Velasco I, Yeo TK, Chen S, et al: Renal lipotoxicity-associated inflammation and insulin resistance affects actin cytoskeleton organization in podocytes. PLoS One 2015; 10: $\mathrm{e} 014229$.

10 Kershaw EE, Flier JS: Adipose tissue as an endocrine organ. J Clin Endocrinol Metab 2004; 89:2548-2556.

11 Berl T, Henrich W: Kidney-heart interactions: epidemiology, pathogenesis, and treatment. Clin J Am Soc Nephrol 2006;1: $8-18$.

12 D'Agati VD, Chagnac A, de Vries AP, Levi M, Porrini E, Herman-Edelstein M, et al: Obesity-related glomerulopathy: clinical and pathologic characteristics and pathogenesis. Nat Rev Nephrol 2016;12:453-471.

13 Ristow M, Müller-Wieland D, Pfeiffer A, Krone W, Kahn CR: Obesity associated with a mutation in a genetic regulator of adipocyte differentiation. N Engl J Med 1998;339:953959.

14 Medina-Gomez G, Yetukuri L, Velagapudi V, Campbell M, Blount M, Jimenez-Linan M, et al: Adaptation and failure of pancreatic beta cells in murine models with different degrees of metabolic syndrome. Dis Model Mech 2009;2:582-592

15 Martínez-García C, Izquierdo A, Velagapudi V, Vivas Y, Velasco I, Campbell M, et al: Ac- celerated renal disease is associated with the development of metabolic syndrome in a glucolipotoxic mouse model. Dis Model Mech 2012;5:636-648.

16 Luzar B, Ferluga D: Role of lipids in the progression of renal disease in systemic lupus erythematosus patients. Wien Klin Wochenschr 2000;112:716-721.

17 Rutkowski JM, Wang ZV, Park AS, Zhang J, Zhang D, Hu MC, et al: Adiponectin promotes functional recovery after podocyte ablation. J Am Soc Nephrol 2013;24:268282.

18 Corrales P, Izquierdo-Lahuerta A, MedinaGómez G: Maintenance of kidney metabolic homeostasis by PPAR gamma. Int J Mol Sci 2018;19:2063-2074.

19 Lee YJ, Han HJ: Troglitazone ameliorates high glucose-induced EMT and dysfunction of SGLTs through PI3K/Akt, GSK-3 $\beta$, Snail1, and $\beta$-catenin in renal proximal tubule cells. Am J Physiol Renal Physiol 2010;298:F1263F1275.

20 Arima S, Kohagura K, Takeuchi K, Taniyama Y, Sugawara A, Ikeda Y, et al: Biphasic vasodilator action of troglitazone on the renal microcirculation. J Am Soc Nephrol 2002;13: 342-349.

21 Benson SC, Pershadsingh HA, Ho CI, Chittiboyina A, Desai P, Pravenec M, et al: Identification of telmisartan as a unique angiotensin II receptor antagonist with selective PPARgamma-modulating activity. Hypertension 2004;43:993-1002. 